\title{
Effect of Material Properties in Certain Thermoelastic Contact Problems
}

\author{
J. R. Barber \\ Mem. ASME \\ and Applied Mechanics, \\ University of Michigan,
}

Department of Mechanical Engineering Ann Arbor, Ml 48109-2125
When two conforming bodies slide against each other, frictional heating and thermoelastic distortion generally causes the contacting surfaces to become convex and hence leads to a reduction in the size of the contact area. It is shown that, under such circumstances, the contact area is independent of the applied contact loads and the thermal and mechanical fields are linearly proportional to these loads. For twodimensional problems involving a plane boundary, it is shown that there is a reduced dependence on material properties and in the case of a single material, the solution depends only on a single parameter which can be interpreted as a dimensionless sliding speed. These results extend to both steady-state and transient problems and therefore also characterize the critical sliding speed above which the system is unstable.

\section{Introduction}

In an interesting series of papers, Dundurs and his co-workers (Dundurs and Stippes, 1970; Dundurs, 1975; Keer et al., 1972) established some unexpectedly simple results for receding unilateral frictionless contact problems; i.e., problems in which the extent of the contact area under load is contained within or identical to that in the unloaded state. In particular, they showed that the contact area may decrease discontinuously as soon as the load is applied, but that further increase of load causes no further change in the contact area. It then follows that the stress and displacement fields are proportional to the applied boundary tractions or displacements as long as these retain the same sign. Furthermore, in plane elasticity, if the boundary conditions are defined in terms of tractions, the stresses are independent of the elastic constants, including Poisson's ratio and there is a reduced dependence on material properties for cases involving dissimilar materials.

Recent numerical studies by the present authors of thermoelastic contact problems involving frictional heating have exhibited a related reduced dependence on material properties. In the present paper, we shall establish the conditions under which such reduced dependence can be expected and draw some interesting conclusions for steady-state and transient thermoelastic contact and for thermoelastic contact stability.

\section{Problem Statement}

We consider the conforming contact problem of Fig. 1(a) in which two plane elastic bodies make contact over an extended region $\Gamma_{0}$ when there is no applied load. We now apply some boundary tractions, as shown in Fig. 1(b), and allow relative sliding to occur between the bodies in the out-of-plane direction, resulting in an out-of-plane shear stress $\left|\sigma_{n z}\right|=f p$, where $n$ is the local normal to the contact surface, $p$ is the contact pressure, and $f$ is the coefficient of friction. If the sliding velocity is $V$, heat will be generated at the interface according to the relation

Contributed by the Applied Mechanics Division of THE AMERICAN SOCIETY of MECHanical ENGINEERS for publication in the ASME JoURNAL OF APPLIED Mechanics.

Discussion on the paper should be addressed to the Technical Editor, Professor Lewis T. Wheeler, Department of Mechanical Engineering, University of Houston, Houston, TX 77204-4792, and will be accepted until four months after final publication of the paper itself in the ASME Journal of APPLIED MECHANICS.

Manuscript received by the ASME Applied Mechanics Division, Sept. 16, 1997; final revision, Apr. 16, 1998. Associate Technical Editor: L. T. Wheeler.

$$
q=f V p
$$

The contact area may change as a result of the thermal and mechanical loading and the instantaneous value is denoted by $\Gamma_{c}$. Heat input to either body produces a locally convex thermoelastic distortion (Dundurs, 1974) and hence the boundary condition (1) generally results in receding contact $\left(\Gamma_{c} \subseteq \Gamma_{0}\right)$.

Problems of this class are of importance because of the application to brakes, clutches, and seals. Burton et al. (1973) and Barber (1976) obtained steady-state solutions for the case of two sliding half-planes and related transient problems have been investigated numerically by Azarkhin and Barber (1986) and Zagrodzki (1990). If the sliding speed $V$ is sufficiently high, both steady-state and transient solutions can be unstable in the sense that an arbitrarily small perturbation in the initial conditions can cause large changes in the subsequent behavior (Dow and Burton, 1972; Du et al., 1997). This phenomenon is known as frictionally excited thermoelastic instability or TEI (Barber, 1969).

2.1 Thermal conditions. The temperature field $T^{i}$ in the bodies must satisfy the heat conduction equation

$$
\nabla^{2} T^{i}=\frac{1}{k_{i}} \frac{\partial T^{i}}{\partial t}
$$

where $i=1,2$ denotes bodies 1,2, respectively, $t$ is time, and $k_{i}$ is the thermal diffusivity of material $i$.

In the contact region, $\Gamma_{c}$, we assume continuity of temperature

$$
T^{1}=T^{2} \quad \text { in } \quad \Gamma_{c}
$$

and a heat balance yields

$$
q^{1}-q^{2}=-K_{1} \frac{\partial T^{1}}{\partial n}+K_{2} \frac{\partial T^{2}}{\partial n}=f V p \quad \text { in } \quad \Gamma_{c}
$$

from Eq. (1), where $K_{i}$ is the thermal conductivity of material $i$ and we have adopted the convention that the common normal $n$ points into body 1 .

At the free boundaries of the body $\Gamma-\Gamma_{0}$, we assume that there is radiation to a medium at a reference temperature of zero and hence

$$
q_{n}=-K_{i} \frac{\partial T^{i}}{\partial n}=h T^{i} \quad \text { in } \quad \Gamma-\Gamma_{0},
$$




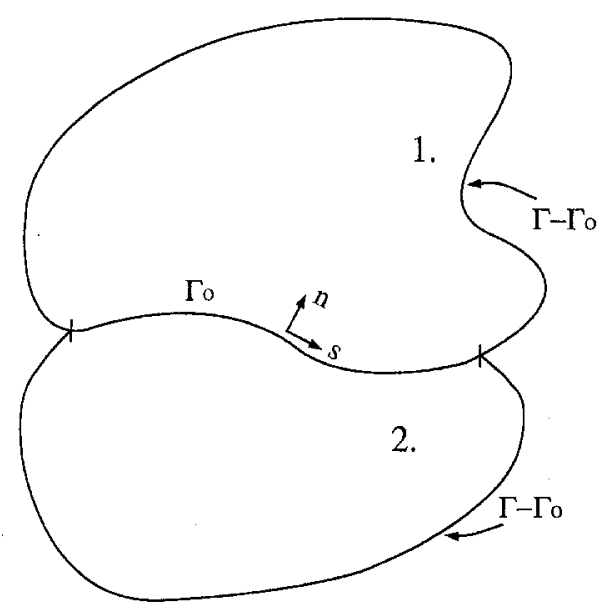

Fig. 1 (a)

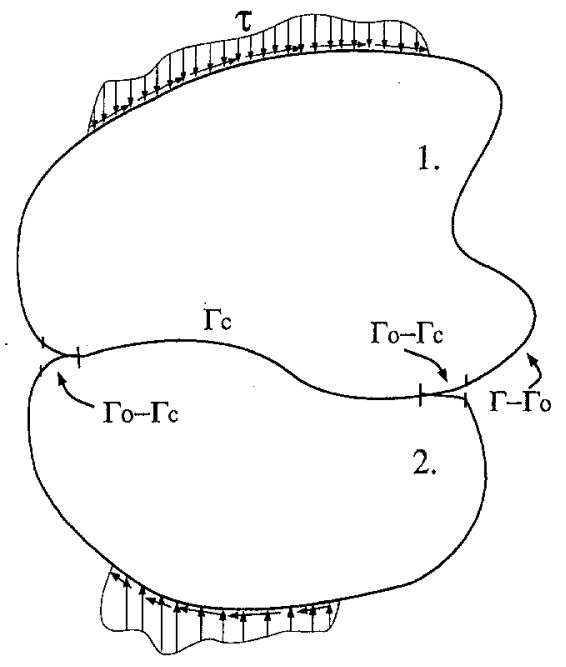

Fig. $1(b)$

Fig. 1 A receding contact problem. (a) With no applied tractions, the contact region is $\Gamma_{0}$. (b) With applied tractions, $\tau$, the contact region is $\Gamma_{c}$, and $\Gamma_{c} \subseteq \Gamma_{0}$.

where $h$ is a heat transfer coefficient. Finally, in the region $\Gamma_{0}$ $-\Gamma_{c}$ which loses contact as a result of thermoelastic distortion and loading, we assume there is heat transfer between the bodies through a heat transfer coefficient $h_{0}$, i.e.,

$$
-K_{1} \frac{\partial T^{1}}{\partial n}=-K_{2} \frac{\partial T^{2}}{\partial n}=h_{0}\left(T^{2}-T^{1}\right) \quad \text { in } \quad \Gamma_{0}-\Gamma_{c} .
$$

We note that by setting $h=0$ or $h_{0}=0$ in (5), (6), we recover the thermally insulated boundary condition, while by setting $h=\infty$ or $h_{0}=\infty$, we obtain the boundary conditions $T$ $=0$ and $T^{\mathrm{L}}=T^{2}$, respectively.

2.2 Mechanical conditions. We assume that there are no body forces-i.e., that the bodies are loaded only on the boundaries $\Gamma$-in which case the stress field in both bodies must satisfy the equilibrium relations

$$
\frac{\partial \sigma_{x x}}{\partial x}+\frac{\partial \sigma_{x y}}{\partial y}=0 ; \quad \frac{\partial \sigma_{y x}}{\partial x}+\frac{\partial \sigma_{y y}}{\partial y}=0
$$

and the resulting strains must satisfy the compatibility equation

$$
\frac{\partial^{2} e_{x x}}{\partial y^{2}}+\frac{\partial^{2} e_{y y}}{\partial x^{2}}=2 \frac{\partial^{2} e_{x y}}{\partial x \partial y} .
$$

These quantities are related by the thermoelastic Hooke's law

$$
\begin{gathered}
e_{x x}^{i}=\frac{\sigma_{x x}^{i}}{2 \mu_{i}}-\frac{\left(3-\kappa_{i}\right)\left(\sigma_{x x}^{i}+\sigma_{y y}^{i}\right)}{8 \mu_{i}}+\eta_{i} T^{i} \\
e_{x y}^{i}=\frac{\sigma_{x y}^{i}}{2 \mu_{i}}
\end{gathered}
$$

etc., where

$$
\begin{gathered}
\kappa_{i}=\left(3-4 \nu_{i}\right) ; \quad \eta_{i}=\gamma_{i}\left(1+\nu_{i}\right) \quad \text { for plane strain, } \\
\kappa_{i}=\frac{\left(3-\nu_{i}\right)}{\left(1+\nu_{i}\right)} ; \quad \eta_{i}=\gamma_{i} \quad \text { for plane stress }
\end{gathered}
$$

and $\mu_{i}, \nu_{i}, \gamma_{i}$ are, respectively, the shear modulus, Poisson's ratio, and coefficient of thermal expansion ${ }^{1}$ for material $i$.

We assume traction boundary conditions on the free boundaries, i.e.

$$
\sigma_{n n}=\tau_{n} ; \quad \sigma_{n s}=\tau_{s} \text { in } \Gamma-\Gamma_{0},
$$

where $n, s$ are Cartesian coordinates normal and tangential to the local boundary and $\tau_{n}, \tau_{s}$ are prescribed normal and tangential tractions, respectively.

In the contact region, there is no in-plane shear traction and the normal traction is continuous, so

$$
\sigma_{n n}^{1}=\sigma_{n n}^{2} \equiv-p ; \quad \sigma_{n s}^{1}=\sigma_{n s}^{2}=0 \text { in } \Gamma_{c} .
$$

In addition, we have the kinematic contact condition

$$
u_{n}^{1}=u_{n}^{2} \text { in } \Gamma_{c}
$$

and the contact pressure is required to be non-negative; i.e.,

$$
p \geq 0 \text { in } \Gamma_{c} .
$$

Finally, the region that loses contact must be traction-free

$$
\sigma_{n n}^{1}=\sigma_{n n}^{2}=0 ; \quad \sigma_{n s}^{1}=\sigma_{n s}^{2}=0 \quad \text { in } \Gamma_{0}-\Gamma_{c}
$$

and the displacements must result in a non-negative gap, giving

$$
u_{n}^{1}-u_{n}^{2} \geq 0 \text { in } \Gamma_{0}-\Gamma_{c} .
$$

We restrict attention to the case where the two bodies are both simply connected ${ }^{2}$, in which case the above conditions completely define the problem of Fig. 1.

\section{Dimensionless Formulation}

In order to demonstrate the dependence of the solution on material parameters, it is convenient to recast these conditions in terms of dimensionless quantities, normalized with respect to the applied tractions. For this purpose, we introduce a loading parameter $S>0$ with units of stress and define dimensionless tractions $\hat{\tau}_{n}, \hat{\tau}_{s}$ through the relations

$$
\tau_{n}=S \hat{\tau}_{n} ; \quad \tau_{s}=S \hat{\tau}_{s} .
$$

We also introduce a geometric length scale $a$ which will enable us to expose simplifications resulting from geometric self-similarity.

With these preliminaries, we now define dimensionless coordinates, stresses, displacements, etc., through the relations

$$
\begin{gathered}
x=a \hat{x} ; \quad y=a \hat{y} ; \quad n=a \hat{n} ; \quad s=a \hat{s} ; \\
t=\frac{a^{2} \hat{t}}{k_{1}}
\end{gathered}
$$

\footnotetext{
${ }^{1}$ Notice that we here use the symbol $\gamma$ rather than the more familiar $\alpha$ for the coefficient of thermal expansion to avoid confusion with Dundurs bimaterial constant $\alpha$ which appears in Section 3.

${ }^{2}$ If either body is multiply connected, the compatibility condition must be supplemented a set of Cesaro integrals around each hole (Love, 1927).
} 


$$
\begin{gathered}
\sigma=S \hat{\sigma} ; \quad p=S \hat{p} ; \\
u^{i}=\frac{a S\left(\kappa_{i}+1\right) \hat{u}^{i}}{\mu_{i}} \\
T^{i}=\frac{S\left(\kappa_{i}+1\right) \hat{T}^{i}}{\mu_{i} \eta_{i}} \\
q^{i}=\frac{S K_{i}\left(\kappa_{i}+1\right) \hat{q}^{i}}{a \mu_{i} \eta_{i}} .
\end{gathered}
$$

We also define the alternate strain measure $\hat{e}^{i}$ through the relation

$$
e^{i}=\frac{S\left(\kappa_{i}+1\right) \hat{e}^{i}}{\mu_{i}} .
$$

In this notation, the governing equations (2), (7) - (10) take the simpler forms

$$
\begin{gathered}
\nabla^{2} \hat{T}^{1}=\frac{\partial \hat{T}^{1}}{\partial \hat{t}} ; \quad \nabla^{2} \hat{T}^{2}=\hat{k} \frac{\partial \hat{T}^{2}}{\partial \hat{t}} \\
\frac{\partial \hat{\sigma}_{x x}}{\partial \hat{x}}+\frac{\partial \hat{\sigma}_{x y}}{\partial \hat{y}}=0 ; \quad \frac{\partial \hat{\sigma}_{y x}}{\partial \hat{x}}+\frac{\partial \hat{\sigma}_{y y}}{\partial \hat{y}}=0 \\
\frac{\partial^{2} \hat{e}_{x x}}{\partial \hat{y}^{2}}+\frac{\partial^{2} \hat{e}_{y y}}{\partial \hat{x}^{2}}=2 \frac{\partial^{2} \hat{e}_{x y}}{\partial \hat{x} \partial \hat{y}} \\
\hat{e}_{x x}^{i}=\frac{\hat{\sigma}_{x x}^{i}}{2\left(\kappa_{i}+1\right)}-\frac{\left(3-\kappa_{i}\right)\left(\hat{\sigma}_{x x}^{i}+\hat{\sigma}_{y y}^{i}\right)}{8\left(\kappa_{i}+1\right)}+\hat{T}^{i} \\
\hat{e}_{x y}^{i}=\frac{\hat{\sigma}_{x y}^{i}}{2\left(\kappa_{i}+1\right)},
\end{gathered}
$$

where

$$
\hat{k}=\frac{k_{1}}{k_{2}}
$$

A more convenient form of the compatibility Eq. (29) can be obtained by substituting for the strains using (30), (31) and using the equilibrium Eq. (28) to eliminate the shear stress terms. After cancelling a nonzero dimensionless parameter, we obtain

$$
\nabla^{2}\left(\hat{\sigma}_{x x}^{i}+\hat{\sigma}_{y y}^{i}+8 \hat{T}^{i}\right)=0 .
$$

The corresponding dimensionless forms of the boundary conditions $(3)-(6),(13)-(18)$ are

$$
\begin{gathered}
\hat{T}^{1}=\hat{\eta} \hat{M} \hat{T}^{2} ; \\
-\frac{\partial \hat{T}^{1}}{\partial \hat{n}}+\frac{\hat{M} \hat{\eta}}{\hat{K}} \frac{\partial \hat{T}^{2}}{\partial \hat{n}}=\hat{V} \hat{p} ; \\
\hat{\sigma}_{m n}^{1}=\hat{\sigma}_{m n}^{2} \equiv-\hat{p} ; \quad \hat{\sigma}_{n s}^{1}=\hat{\sigma}_{n s}^{2}=0 ; \\
\hat{u}_{n}^{1}=\hat{M} \hat{u}_{n}^{2} ; \\
\hat{p} \geq 0,
\end{gathered}
$$

in $\Gamma_{c}$,

$$
\begin{gathered}
-\frac{\partial \hat{T}^{1}}{\partial \hat{n}}=\hat{h} \hat{T}^{1} ; \quad-\frac{\partial \hat{T}^{2}}{\partial \hat{n}}=\hat{K} \hat{h} \hat{T}^{2} ; \\
\hat{\sigma}_{n n}=\hat{\tau}_{n} ; \quad \hat{\sigma}_{n s}=\hat{\tau}_{s},
\end{gathered}
$$

in $\Gamma-\Gamma_{0}$ and

$$
\begin{gathered}
-\frac{\partial \hat{T}^{\prime}}{\partial \hat{n}}=-\frac{\hat{M} \hat{\eta}}{\hat{K}} \frac{\partial \hat{T}^{2}}{\partial \hat{n}}=\hat{h}_{0}\left(\hat{M} \hat{\eta}^{2}-\hat{T}^{\mathrm{l}}\right) ; \\
\hat{\sigma}_{m}^{1}=\hat{\sigma}_{m}^{2}=0 ; \quad \hat{\sigma}_{n s}^{1}=\hat{\sigma}_{n, s}^{2}=0 ;
\end{gathered}
$$

$$
\hat{u}_{n}^{1}-\hat{M} \hat{u}_{n}^{2} \geq 0
$$

in $\Gamma_{0}-\Gamma_{c}$, where

$$
\begin{gathered}
\hat{\eta}=\frac{\eta_{1}}{\eta_{2}} ; \quad \hat{M}=\frac{\mu_{1}\left(\kappa_{2}+1\right)}{\mu_{2}\left(\kappa_{1}+1\right)} ; \quad \hat{K}=\frac{K_{1}}{K_{2}} ; \\
\hat{h}=\frac{h a}{K_{1}} ; \quad \hat{h}_{0}=\frac{h_{0} a}{K_{1}}
\end{gathered}
$$

and

$$
\hat{V}=\frac{f V \mu_{1} \eta_{1} a}{K_{1}\left(\kappa_{1}+1\right)}
$$

Notice that $\hat{M}$ can be defined in terms of Dundurs bimaterial parameter $\alpha$ through

$$
\hat{M}=\frac{1-\alpha}{1+\alpha} .
$$

It follows that in the dimensionless formulation, the problem depends on the material properties only through $\kappa_{i}$ and the dimensionless parameters defined in Eqs. (32), (44), (45).

\section{Linearity With Loading Parameter}

The loading parameter $S$ does not appear in the dimensionless formulation of the problem as defined by the above equations. It follows immediately that the extent of the contact area $\Gamma_{c}$, which is determined by the inequalities (38), (43), is independent of $S$. Furthermore, since the only nonlinearity in the problem is that associated with these inequalities and the possibility of the contact area varying with load, it then follows that the temperature, stress, and displacement fields are all linear with $S$.

It must be emphasised that this result applies only for the case where $S$ is positive, since in deriving the inequalities (38), (43) from (16), (18) we have cancelled a common factor $S$. However, this is not a serious restriction since with an appropriate sign convention the case $S<0$ will correspond to loading that would not support contact even without thermoelastic effects.

Notice that these results apply to transient as well as steadystate problems. Thus, if the bodies are initially at temperature $T=0$ (for example) and sliding starts at time $t=0$, we anticipate that the contact area will start at some value $\Gamma_{c}(0) \leq \Gamma_{0}$ and will get progressively smaller as thermal distortion occurs. The fact that the dimensionless formulation does not contain $S$ demonstrates that the evolution of the contact area in time, $\Gamma_{c}(\hat{t})$, is independent of $S$ and that the instantaneous temperature, displacement, and stress fields for different values of $S$ will be linear scalings of each other for a particular value of $\hat{t}$.

The same argument applies if the dimensionless tractions $\hat{\tau}_{n}, \hat{\tau}_{s}$ and/or the sliding speed $\hat{V}$ are functions of time.

We have developed Eqs. (27) - (43) for the simpler twodimensional case, but it is clear that a similar line of reasoning could be developed for the three-dimensional problem of Fig. 2 , in which a brake pad slides against a rotating annular disk.

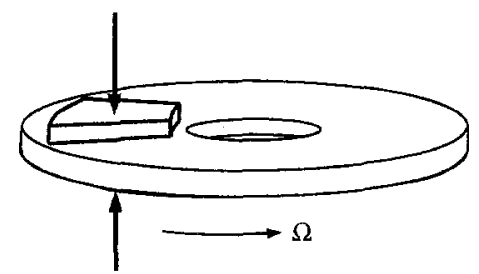

Fig. 2 A three-dimensional receding contact problem for a disk brake. As in the two-dimensional case, the contact area will be independent of the applied load. 
In addition, the argument is readily extended to cases involving multiply connected regions and/or body force loading. To see this, suppose that a solution has been obtained for a particular value of $S\left(=S_{1}\right)$, so that the dimensional temperature, displacement, and stress fields are all known and in particular that they satisfy the unilateral contact inequalities analogous to $(16,18)$ in $\Gamma_{0}-\Gamma_{c}(t)$ at all times $t$. We now postulate that the corresponding fields for a different but proportional loading scenario $S=S_{2}$ will be $S_{2} / S_{1}$ times the original solution. Clearly all the linear governing equations and boundary conditions will be satisfied by this assumption and the new inequality constraints will simply be a positive multiplier of the old ones and hence will also still be satisfied.

We conclude therefore that if a brake pad conforms to the disk at zero applied load, the evolution of the contact area due to combined elastic and thermoelastic effects will be independent of the loading parameter.

This result leads to a remarkable conclusion in the case of mechanical seals. Burton et al. (1973) showed that if a seal between two elastic half-planes operates above the critical speed, the system will be unstable, but will tend to a stable nonuniform steady state in which there are regions of contact and separation, permitting leakage to occur through the separation regions. If the applied load is increased in a misguided attempt to reestablish full contact, the extent of the separation areas will be unchanged, but all the thermal and mechanical fields will be increased, leading in particular to an increased gap and hence increased leakage. The original proof by Burton et al. (1973) was restricted to their particular system, but it is clear from the arguments of this section that the result is of completely general application.

Notice that the three-dimensional argument depends on the unloaded contact problem being conforming in all kinematic configurations. For this to occur, all parts of the contacting surfaces must have the same shape and this restricts the result to cases where the contacting surfaces are plane or (possibly incomplete) surfaces of revolution (e.g., a drum brake). No such restriction applies in the two-dimensional case, since the motion then occurs out-of-plane.

\section{The Contact Boundary Conditions}

We now return to the two-dimensional formulation of Eqs. (27) - (43) and consider in particular the contact boundary conditions (37), (43). Under certain circumstances Eq. (37) can be replaced by a relationship involving strains only. Dundurs (1989) showed that the change $\Delta \hat{C}$ in the curvature of an interface can be written in terms of strains as

$$
\Delta \hat{C}=2 \frac{\partial \hat{e}_{s n}}{\partial s}-\frac{\partial \hat{e}_{s s}}{\partial n}-\hat{C} \hat{e}_{n n}
$$

where the dimensionless curvature $\hat{C}$ is defined such that $\hat{C} \hat{\mathbf{s}}=$ $-\partial \hat{\mathbf{n}} / \partial \hat{s}$. Markenscoff and Wheeler (1996) showed that the contact condition (37) implies that

$$
\Delta \hat{C}^{1}-\Delta \hat{C}^{2}=\hat{C}^{\prime}\left(\hat{u}_{s}^{1}-\hat{u}_{s}^{2}\right) \text { in } \Gamma_{c} .
$$

They were also able to express this condition in terms of strains only in the form

$$
\frac{d}{d \hat{s}}\left(\frac{\Delta \hat{C}^{1}-\Delta \hat{C}^{2}}{\hat{C}^{\prime}}\right)=\hat{e}_{s s}^{1}-\hat{e}_{s s}^{2} \text { in } \Gamma_{c} .
$$

In the present problem, $\hat{\sigma}_{n s}^{i}$ and hence $\hat{e}_{n s}^{i}$ are zero throughout $\Gamma_{0}$, from (36), (42), so (47) simplifies to

$$
\Delta \hat{C}=-\frac{\partial \hat{e}_{s s}}{\partial n}-\hat{C} \hat{e}_{n n} \text { in } \Gamma_{0}
$$

Substituting for the strains from (30), we have

$$
\begin{array}{r}
\Delta \hat{C}^{i}=-\frac{1}{2\left(\kappa^{i}+1\right)} \frac{\partial \hat{\sigma}_{s s}^{i}}{\partial \hat{n}}+\frac{\left(3-\kappa^{i}\right)}{8\left(\kappa^{i}+1\right)}\left(\frac{\partial \hat{\sigma}_{s s}^{i}}{\partial \hat{n}}+\frac{\partial \hat{\sigma}_{n n}^{i}}{\partial \hat{n}}\right) \\
-\frac{\partial \hat{T}^{i}}{\partial \hat{n}}-\hat{C}\left(\frac{\hat{\sigma}_{m n}^{i}}{2\left(\kappa^{i}+1\right)}-\frac{\left(3-\kappa^{i}\right)}{8\left(\kappa^{i}+1\right)}\right. \\
\left.\times\left(\hat{\sigma}_{s s}^{i}+\hat{\sigma}_{m n}^{i}\right)+\hat{T}^{i}\right) \text { in } \Gamma_{0} .
\end{array}
$$

This expression can be simplified using the equilibrium equation

$$
\frac{\partial \hat{\sigma}_{n n}^{i}}{\partial \hat{n}}=-\frac{\partial \hat{\sigma}_{n s}^{i}}{\partial \hat{s}}-\hat{C}\left(\hat{\sigma}_{s s}^{i}-\hat{\sigma}_{n n}^{i}\right)
$$

(see, for example, Dundurs (1989), Eq. (21)). Substituting (52) into (51) and noting once more that $\hat{\sigma}_{n s}^{i}=0$ in $\Gamma_{0}$, we obtain

$$
\begin{aligned}
\Delta \hat{C}^{i}=-\frac{1}{8} \frac{\partial \hat{\sigma}_{s s}^{i}}{\partial \hat{n}}- & \frac{\partial \hat{T}^{i}}{\partial \hat{n}} \\
& +\hat{C}\left(\frac{\left(2-\kappa^{i}\right) \hat{\sigma}_{n n}^{i}}{8\left(\kappa^{i}+1\right)}-\hat{T}^{i}\right) \text { in } \Gamma_{0} .
\end{aligned}
$$

\section{Contact Along a Straight Boundary}

In general, the terms involving $\hat{C}$ in Eq. (53) will introduce a dependence on $\kappa^{i}$ into the problem. However, for the special case where the initial contact boundary is straight, $\hat{C}=0$ and (53) reduces to

$$
\Delta \hat{C}^{i}=\frac{\partial^{2} \hat{u}_{n}^{i}}{\partial \hat{s}^{2}}=-\frac{1}{8} \frac{\partial \hat{\sigma}_{s s}^{i}}{\partial \hat{n}}-\frac{\partial \hat{T}^{i}}{\partial \hat{n}} \text { in } \Gamma_{0} .
$$

We can therefore integrate along the straight line $\Gamma_{0}$ to obtain

$$
\begin{aligned}
\hat{u}_{n}^{1}-\hat{M} \hat{u}_{n}^{2}=-\iint\left(\frac { 1 } { 8 } \left[\frac{\partial \hat{\sigma}_{s s}^{1}}{\partial \hat{n}}\right.\right. & \left.+\hat{M} \frac{\partial \hat{\sigma}_{s s}^{2}}{\partial \hat{n}}\right] \\
+ & {\left.\left[\frac{\partial \hat{T}^{1}}{\partial \hat{n}}+\hat{M} \frac{\partial \hat{T}^{2}}{\partial \hat{n}}\right]\right) d s d s }
\end{aligned}
$$

in $\Gamma_{0}$. The integral in (55) will contain two arbitrary constants of integration corresponding to rigid-body translation and rotation.

If we use (55) to substitute for $\hat{u}_{n}^{1}-\hat{M} \hat{u}_{n}^{2}$ in (37), (43), the entire problem of Fig. 1 can be stated in terms of the two functions $\hat{T}, \hat{\sigma}^{i}$, using the boundary conditions (34)-(43) and the governing Eqs. (27), (28), (33). It follows that the solution depends upon the material properties only through the dimensionless parameters $\hat{M}, \hat{\eta}, \hat{K}, \hat{k}, \hat{h}, \hat{h}, \hat{V}$. In the steady-state problem, there is no dependence on $\hat{k}$ and if the thermal boundary conditions in $\Gamma-\Gamma_{c}$ are all of the form $q=0$ or $T=0$, the dependence on material properties is further reduced to the set $\hat{M}, \hat{\eta}, \hat{K}, \hat{V}$.

6.1 Similar Materials. If the two materials are similar, we have $\hat{M}=\hat{\eta}=\hat{K}=\hat{k}=1$ and the problem depends only on $\hat{h}, \hat{h}_{c}, \hat{V}$. Remarkably, this reduces to dependence on the single dimensionless group $\hat{V}$ in the case where the thermal boundary conditions in $\Gamma-\Gamma_{c}$ are all of the form $q=0$ or $T$ $=0$. This remains true in the case of transient problems.

6.2 One Material a Rigid Nonconductor. If material 2 is a rigid nonconductor, we have $\hat{M}=0, \hat{\eta}=\hat{K}=\hat{k}=\infty$ and once again the problem depends on the single parameter $\hat{V}$ for homogeneous temperature or heat flux boundary conditions in $\Gamma-\Gamma_{c}$, 
6.3 Thermoelastic Contact Stability. Dow and Burton (1972) were the first to demonstrate that a problem defined by the frictional heating boundary condition (1) would be unstable if the sliding speed exceeded a certain critical value. They also pioneered a perturbation method for determining the critical speed $V_{0}$ in which they examine the conditions under which a small perturbation in the stress and temperature fields can grow exponentially with time. This method has since been used extensively for determining critical speed in more complex systems, both in analytical and numerical implementations (see, for example, Lee and Barber, 1993; Du et al., 1997).

Burton's perturbation method is essentially equivalent to a solution of the transient thermoelastic contact problem of Fig. 1 -indeed, the general transient problem can in principle be written down as an eigenfunction expansion using the eigenmodes of Burton's solution. Thus, the above arguments carry over to the stability of any solutions obtained. In particular, we conclude that if two bodies of similar materials make contact at a plane boundary or one body makes contact with a rigid nonconducting plane surface, the stability of a given steadystate or transient solution can be defined in terms of a single dimensionless critical speed

$$
\hat{V}_{0}=\frac{f V_{0} \mu \eta a}{K(\kappa+1)},
$$

for homogeneous temperature or heat flux boundary conditions in $\Gamma-\Gamma_{c}$.

This result is of significant value in the design of sliding systems against thermoelastic instability. In many cases, the design configuration is too complex to make a full numerical solution of the problem practicable, but using equation (56), we can conclude that the critical speed will be increased (and hence the system made more stable) by any change in material properties or dimensions that reduces the value of $\hat{V}_{0}$ for given $V_{0}$; i.e., that reduces

$$
\frac{f \mu \eta a}{K(\kappa+1)}
$$

\section{Conclusions}

We have demonstrated that the extent of the contact area $\Gamma_{c}$ in the thermoelastic contact problem of Fig. 1 remains invariant under proportional loading if $\Gamma_{c} \subseteq \Gamma_{0}$; i.e., if the final contact area is included within that occurring at zero applied load. It then also follows that the temperature, stress, and displacement fields are all linear with the applied tractions.

For the two-dimensional problem with sliding at a common straight boundary, we have established a reduction in the depen- dence of the solution on the properties of the materials. In particular, we find that for problems involving two similar materials or the contact of a single deformable body against a rigid plane surface, the solution depends only on a single parameter, which is a dimensionless sliding speed $\hat{V}$. The stability of the solution is also determined by this parameter.

Since most practical problems of this class require numerical solution, these results extend the generality of such solutions and permit conclusions to be drawn about the effect of changes in material properties on stability behavior.

\section{Acknowledgments}

The authors are pleased to acknowledge support from the Ford Motor Company and from the National Science Foundation under contract number CMS-9619527. They are also grateful to Mr. M. Ciavarella of the Politecnico di Bari for some helpful contributions to the calculations in Section 5.

\section{References}

Azarkhin, A., and Barber, J. R., 1986, "Thermoelastic Instability for the Transient Contact Problem of Two Sliding Half-Planes," ASME JouRNAl OF APPLIED MECHANICS, Vol. 53, pp. 565-572.

Barber, J. R., 1969, "Thermoelastic instabilities in the sliding of conforming solids," Proc. Roy. Soc., Lond., Vol. A312, pp. 381-394.

Barber, J. R., 1976, "Some thermoelastic contact problems involving frictional heating,"' $Q$. J. Mech. Appl. Math., Vol. 29, pp. 1-13.

Burton, R. A., Kilaparti, S. R., and Nerlikar, V., 1973, "A limiting stationary configuration with partially contacting surfaces," Wear, Vol. 24, pp. 199-206.

Dow, T. A., and Burton, R. A., 1972, "Thermoelastic instability of sliding contact in the absence of wear," Wear, Vol, 19, pp. 315-328.

Du, Shuqin, Zagrodzki, P., Barber, J. R., and Hulbert, G. M., 1997, "Finite element analysis of frictionally-excited thermoelastic instability," $J$. Thermal Stresses, Vol. 20, pp. 185-201.

Dundurs, J., 1974, "Distortion of a body caused by free thermal expansion," Mech. Res. Comm., Vol. 1, pp. 121-124.

Dundurs, J., 1975, "Properties of elastic bodies in contact," The Mechanics of the Contact between Deformable bodies, A. D. de Pater and J. J. Kalker eds., Delft University Press, pp. 54-66.

Dundurs, J., 1989, "Cavities vis-à-vis Rigid Inclusions and Some Related General Results in Plane Elasticity," ASME Journal of Applied Mechanics, Vol. 56, pp. 786-790.

Dundurs, J., and Stippes, M., 1970, "Role of Elastic Constants in Certain Contact Problems," ASME Journal of APPLIEd Mechanics, Vol. 37, pp. 965 970 .

Keer, L. M., Dundurs, J., and Tsai, K. C., 1972, "Problems Involving a Receding Contact Between a Layer and a Half-Space," ASME JourNAL OF APPLIED Mechanics, Vol. 39, pp. 1115-1120.

Lee, Kwangjin, and Barber, J. R., 1993, "Frictionally-Excited Thermoelastic Instability in Automotive Disk Brakes," ASME Journal of Tribology, Vol. 115, pp. $607-614$.

Love, A. E. H., 1927, A Treatise on the Mathematical Theory of Elasticity, 4th Ed., Cambridge University Press, Cambridge, UK, Section 156A.

Markenscoff, X., and Wheeler, L., 1996, "On conditions at an interface between two materials in plane deformation," J. Elasticity, Vol. 45, pp. 33-44.

Zagrodzki, P., 1990, "Analysis of thermomechanical phenomena in multidisc clutches and brakes," Wear, Vol. 140, pp. 291-308. 